\title{
Extracting the emitter orientation in organic light-emitting diodes from external quantum efficiency measurements
}

Cite as: Appl. Phys. Lett. 105, 043302 (2014); https://doi.org/10.1063/1.4891680

Submitted: 27 June 2014 . Accepted: 18 July 2014 . Published Online: 29 July 2014

Tobias D. Schmidt, Lukas J. Reichardt, Andreas F. Rausch, Sebastian Wehrmeister, Bert J. Scholz, Christian Mayr, Thomas Wehlus, Rossá Mac Ciarnáin, Norbert Danz, Thilo C. G. Reusch, and Wolfgang Brütting

\section{ARTICLES YOU MAY BE INTERESTED IN}

Determination of molecular dipole orientation in doped fluorescent organic thin films by photoluminescence measurements

Applied Physics Letters 96, 073302 (2010); https://doi.org/10.1063/1.3309705

Evidence for non-isotropic emitter orientation in a red phosphorescent organic light-emitting diode and its implications for determining the emitter's radiative quantum efficiency

Applied Physics Letters 99, 163302 (2011); https://doi.org/10.1063/1.3653475

Light extraction and optical loss mechanisms in organic light-emitting diodes: Influence of the emitter quantum efficiency

Journal of Applied Physics 104, 123109 (2008); https://doi.org/10.1063/1.3043800

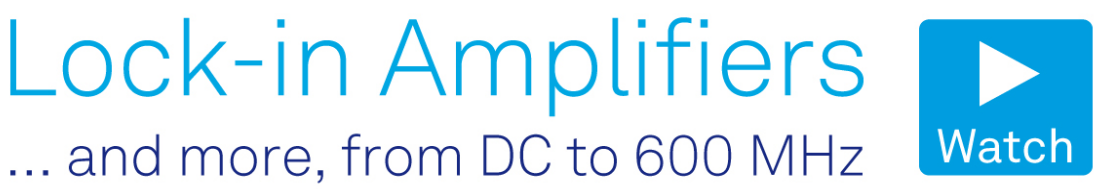




\title{
Extracting the emitter orientation in organic light-emitting diodes from external quantum efficiency measurements
}

\author{
Tobias D. Schmidt, $\left.{ }^{1}{ }^{1, a}\right)$ Lukas J. Reichardt, ${ }^{1}$ Andreas F. Rausch, ${ }^{2}$ Sebastian Wehrmeister, ${ }^{1}$ \\ Bert J. Scholz, ${ }^{1}$ Christian Mayr, ${ }^{1}$ Thomas Wehlus, ${ }^{2}$ Rossá Mac Ciarnáin, ${ }^{3}$ Norbert Danz, ${ }^{3}$ \\ Thilo C. G. Reusch, ${ }^{2}$ and Wolfgang Brütting ${ }^{1, b)}$ \\ ${ }^{1}$ Institute of Physics, University of Augsburg, 86135 Augsburg, Germany \\ ${ }^{2}$ OSRAM OLED GmbH, Wernerwerkstrasse 2, 93049 Regensburg, Germany \\ ${ }^{3}$ Fraunhofer Institute for Applied Optics and Precision Engineering, 07745 Jena, Germany
}

(Received 27 June 2014; accepted 18 July 2014; published online 29 July 2014)

\begin{abstract}
Emitter orientation will play a major role in future applications of organic light-emitting diodes due to its strong impact on the efficiency of the devices. Up to now, determining the orientation of transition dipole moments required elaborate angular-dependent measurements of the light emission pattern. In this paper, we present a simplified and straightforward method to extract the emitter orientation from external quantum efficiency measurements. We demonstrate the validity of the method on three different dye-doped emitting systems. (C) 2014 AIP Publishing LLC.

[http://dx.doi.org/10.1063/1.4891680]
\end{abstract}

Tremendous progress has been made with organic lightemitting diodes (OLEDs) over the last few years. Starting with around $1 \%$ external quantum efficiency in the late $1980 \mathrm{~s},{ }^{1}$ nowadays this value has been easily increased by a factor of $30 .^{2,3}$ Hence, OLEDs are already used in first commercial applications, particularly for mobile displays but also increasingly for large area general lighting elements ${ }^{4}$ and television screens. However, the efficiency and the longterm stability during electrical operation are not yet fully satisfactory. Hence, concepts for low-cost and simple efficiency enhancements which could lead directly to an additional increase in the operational lifetime of the devices, e.g., due to higher light outcoupling, are required.

One promising approach of increasing the light outcoupling efficiency of the devices relies on using emitting guest/ host systems which comprise non-isotropic orientation of their transition dipole moments. A couple of dye-doped emitting systems, fluorescent as well as phosphorescent ones, showing a predominantly horizontal orientation of emissive dipole moments (which is the favorable one) are already known, and the search for new emitter materials and methods influencing the emitter orientation is in progress. However, a direct measure of the orientation of the transition dipole moments of the emitting molecules is not straightforward. On the one hand, investigations of the optical constants of the emitting system by, e.g., ellipsometric methods probably cannot distinguish between the matrix and emitter. On the other hand, the analysis of the angular dependent emission patterns of electrically driven complete OLED stacks needs special emitter to cathode distances, ${ }^{5}$ and photoluminescence measurements of simplified structures without metal layers ${ }^{6}$ need exact adjustments and extensive equipment.

Thus, this Letter presents an alternative approach, namely, determining the orientation of the transition dipole

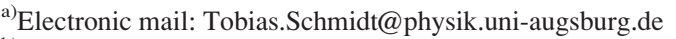

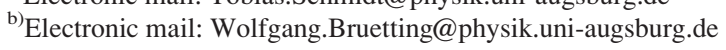

moments of the emitting species in organic light-emitting guest/host systems via simple external quantum efficiency measurements.

The external quantum efficiency (EQE) of organic lightemitting diodes is given by four independent factors ${ }^{7}$

$$
\mathrm{EQE}=\gamma \times \eta_{\mathrm{r}} \times q_{\mathrm{eff}}(q) \times \eta_{\text {out }}=\eta_{\text {int }} \times \eta_{\text {out }} .
$$

Therein, $\gamma$ represents the charge carrier balance. $\eta_{\mathrm{r}}$ accounts for the fraction of created excitons that is quantummechanically allowed to decay under creation of a photon. Thus, $\eta_{\mathrm{r}}$ becomes unity for phosphorescent emitters ${ }^{8-10}$ and can clearly exceed the spin-statistical limit of $25 \%^{11-19}$ of the fluorescent case. The third factor stands for the effective radiative quantum efficiency of the emitting system and depends on its intrinsic value $q$ that is modified by the Purcell effect inside the microcavity-like structure. The last factor gives the outcoupling efficiency of the device and takes into account that not all photons created inside the OLED can contribute to light-emission to the outside world. This factor is strongly influenced by the refractive indices and thicknesses of all used organic and inorganic layers and the orientation of the transition dipole moments of the emitting species. ${ }^{18,20-24}$ The first three factors, namely, the charge carrier balance, the radiative exciton fraction, and the effective radiative quantum efficiency can be combined leading to the so-called internal quantum efficiency $\eta_{\text {int }}$.

Previous publications have pointed out the importance of including substrate mode emission for consistent efficiency analysis in terms of the radiative quantum efficiency of the emitting system as well as the radiative exciton fraction for fluorescent devices exhibiting triplet-to-singlet upconversion. ${ }^{18,20-22,24}$ The strong influence of emitter orientation on the outcoupling efficiency of OLEDs, with and without a macroextractor that gives access to captured substrate modes, allows the investigation of the alignment of the emissive dipoles by simple EQE measurements. This is possible, because the internal quantum efficiency of the device is not modified by attaching a macroextractor to the OLED 

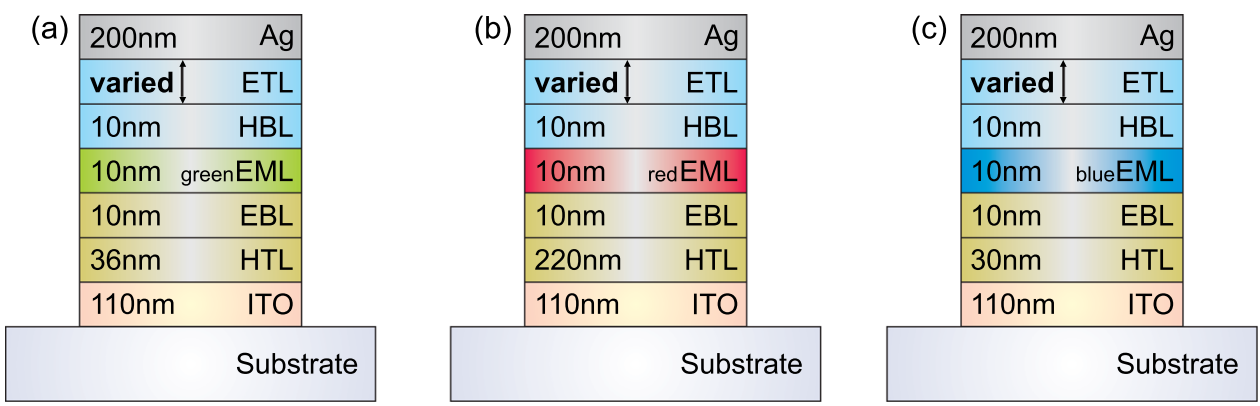

FIG. 1. OLED stack layout for the three different emitting systems with varying ETL thicknesses comprising (a) green phosphorescent ( $q=0.5, \theta=0.33$ ), (b) red phosphorescent $(q=0.7, \theta=0.25)$, and (c) blue fluorescent $(q=0.45, \theta=0.09)$ emitting systems.

substrate. Thus, the ratio of the EQEs measured in an integrating sphere without $\left(\mathrm{EQE}_{\text {direct }}\right)$ and with macroextractor $\left(\mathrm{EQE}_{\text {macro }}\right)$ only depends on the respective light outcoupling factors, which in turn depend on emitter orientation.

Numerical simulations of the power dissipation to the optical modes of an OLED treat the excited molecules as classical electrical dipoles based on a transfer matrix formulation, as described in detail elsewhere. ${ }^{22,24}$ They result in a unique ratio of the direct emission to the emission with extracted substrate modes as a function of the orientation parameter $\theta$. It is defined as the ratio of vertically oriented (with respect to the substrate plane) to the total amount of present transition dipole moments ${ }^{21}$

$$
\theta=\frac{p_{z}}{p_{x}+p_{y}+p_{z}}=\frac{p_{\perp}}{p_{\|}+p_{\perp}} .
$$

Performing these simulations for a set of OLEDs exhibiting different electron transporting layer (ETL) thicknesses, i.e., by varying the distance of the emitting system to the highly reflecting metallic cathode, allows for an analysis of the transition dipole orientation. From the experimental point of view, one simply needs to perform EQE measurements in an integrating sphere with and without a macroextractor (an index-matched half sphere lens attached to the glass substrate) on a series of OLEDs with systematically varied ETL thickness.

Figure 1(a)-1(c) display the stack layouts of three different OLEDs exhibiting an ETL variation (nine devices for each color) with phosphorescent green ${ }^{25}$ and $\operatorname{red}^{21,23}$ as well as fluorescent blue ${ }^{18}$ emitting systems. The used phosphorescent emitting molecules are the well-known Tris[2-phenylpyridinato-C2,N]iridium(III) (Ir(ppy) ${ }_{3}$; green emission, isotropic orientation) doped with 5 wt. \% in a TMM-004 (Merck OLED Materials $\mathrm{GmbH}$ ) matrix and Iridium(III)bis(2-methyldibenzo-[f,h]quinoxaline)acetylacetonate $\left(\operatorname{Ir}(\mathrm{MDQ})_{2}(\mathrm{acac})\right)$; red emission, slight horizontal orientation) doped with $8 \mathrm{wt} . \%$ in an $\mathrm{N}, \mathrm{N}^{\prime}$-bis(naphthalen-1-yl)-N, $\mathrm{N}^{\prime}$-bis(phenyl)benzidine $(\alpha$ NPD) matrix, while the blue fluorescent emitter exhibits nearly completely horizontal orientation. The emission peak wavelengths of the blue, green and red emitter are located at around 470, 520, and $610 \mathrm{~nm}$, respectively. The electron blocking layer (EBL) for all three devices consists of $\alpha$-NPD, while TMM-004 was used as the hole blocking layer (HBL) for the green and blue OLEDs and NET-05 (Novaled GmbH) for the red OLEDs, respectively. Both the ETL and the hole transport layer (HTL) are conductivity doped materials, which cannot be disclosed here. However, their optical constants relevant for the simulations are given in the supporting information. ${ }^{26}$ All three sets of OLEDs, which are presented in this paper, have already been published in terms of efficiency analysis, e.g., by determining the orientation of the transition dipole moments, the radiative quantum efficiency and the radiative exciton fraction. ${ }^{18,21,23,25}$

Figure 2 shows simulations of the ratio of the external quantum efficiency with extracted substrate modes $\left(\mathrm{EQE}_{\text {macro }}\right)$ to the direct emission $\left(\mathrm{EQE}_{\text {direct }}\right)$ for different emitter orientations (from horizontal to vertical) for the red phosphorescent OLED stack depicted in Fig. 1(b) as a function of the ETL thickness. It is clearly visible that the behavior of the EQE ratio strongly depends on the orientation of the transition dipole moments used for the simulations. Particularly, there are ETL thicknesses for which the ratio is very sensitive to horizontal orientation and other ETL thicknesses where vertically oriented emitters show a huge modulation of the EQE ratio. Interestingly, for the ETL thickness where the first cavity minimum occurs $(\sim 155 \mathrm{~nm})$, the influence of horizontal emitter orientation is very pronounced while the sensitivity for vertically oriented emitters is highest in the second cavity maximum $(\sim 250 \mathrm{~nm})$. This behavior can be explained by orientation dependent changes of the energy dissipation to the optical modes, i.e., direct emission

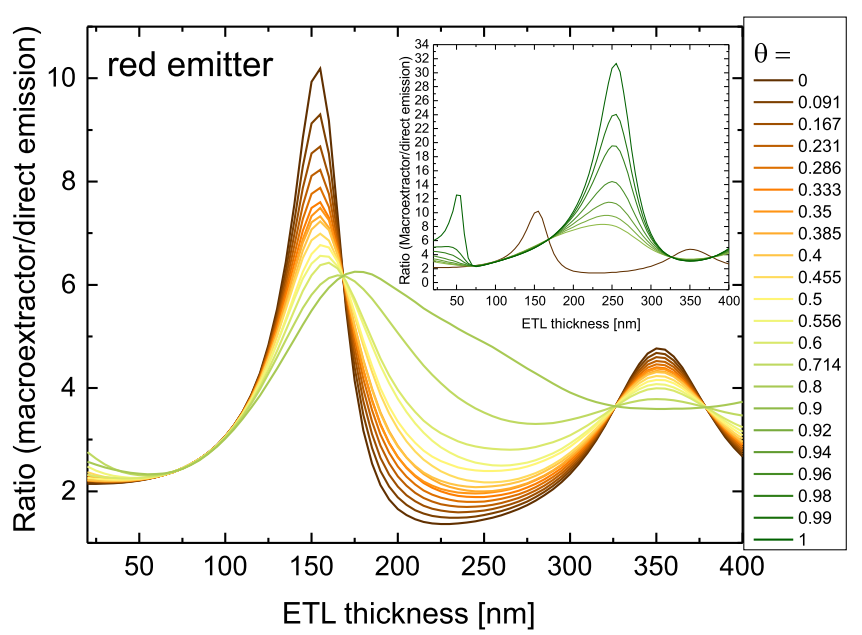

FIG. 2. Simulation of the EQE ratio with and without macroextractor as a function of the ETL thickness of the red OLED stack for different emitter orientations $\theta$. For better visualization, the simulations for vertical orientation $(\theta \geq 0.9)$ together with total horizontal orientation are presented in the inset. 
and substrate modes, of the OLED stack for these special ETL thicknesses. ${ }^{20,22,24}$ With all this information together, it should be possible to determine or validate the orientation of the transition dipole moments of the emitting guest/host system via simple external quantum efficiency measurements for a set of samples comprising different ETL layer thicknesses. Since the emission at the cavity minimum for horizontally oriented dipoles is very weak, high current densities should be used for the investigation. However, by reasoning that the internal quantum efficiency is eliminated by dividing both EQE values (with and without macroextractor) by each other, effects such as current induced quenching, shifts of the emission zone, and charge carrier imbalance as well as possible changes of the radiative exciton fraction by thermally activated triplet-to-singlet up-conversion or exciton density dependent triplet-triplet-annihilation do not affect the analysis.

To validate the reasoning above, Figure 3 clearly demonstrates the constant behavior of the $\mathrm{EQE}$ ratio $\left(\mathrm{EQE}_{\text {macro }} /\right.$ $\left.\left(\mathrm{EQE}_{\text {direct }}\right)\right)$ for different ETL thicknesses with varying current density for OLEDs with the green $\operatorname{Ir}(\mathrm{ppy})_{3}$ emitter. Only the device with the cavity minimum condition $\left(d_{\mathrm{ETL}} \sim 127 \mathrm{~nm}\right)$ shows slight changes of the EQE ratio due to the weak outcoupling efficiency for direct emission and hence somewhat noisy results. Nevertheless, the data confirm that the EQE ratio is not affected by efficiency roll-off at high current densities.

Now, as proof of concept, the three different sample types with varying emitting systems are analyzed in terms of external quantum efficiency measurements with and without macroextractor (half sphere lens). In order to extract the transition dipole orientation, the measured EQE ratios for the green, red, and blue emitting systems together with simulations for varying orientation factors are depicted in Figs. 4(a)-4(c), respectively. All experimental data sets are in good agreement with simulations using the previously published orientation factors $\theta_{\text {green }}=0.33,{ }^{24} \theta_{\text {red }}=0.23,{ }^{21,23}$ and $\theta_{\text {blue }}=0.09^{18}$ determined by both angular dependent photoluminescence and electroluminescence emission pattern analysis. For reference, simulations for deviating

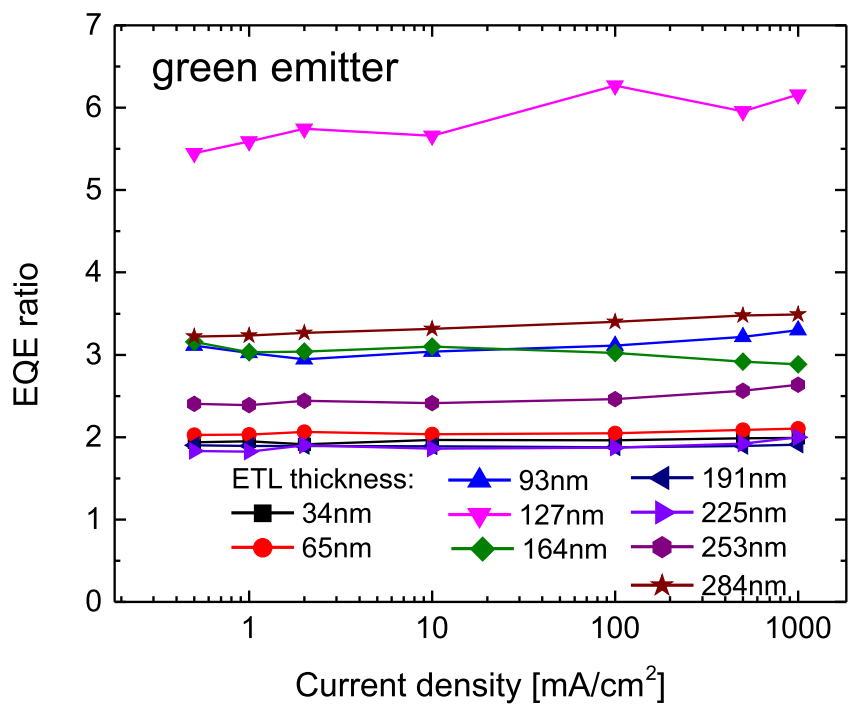

FIG. 3. Measured EQE ratio for the green emitting system for various current densities from 0.5 to $1000 \mathrm{~mA} / \mathrm{cm}^{2}$ for different ETL thicknesses.
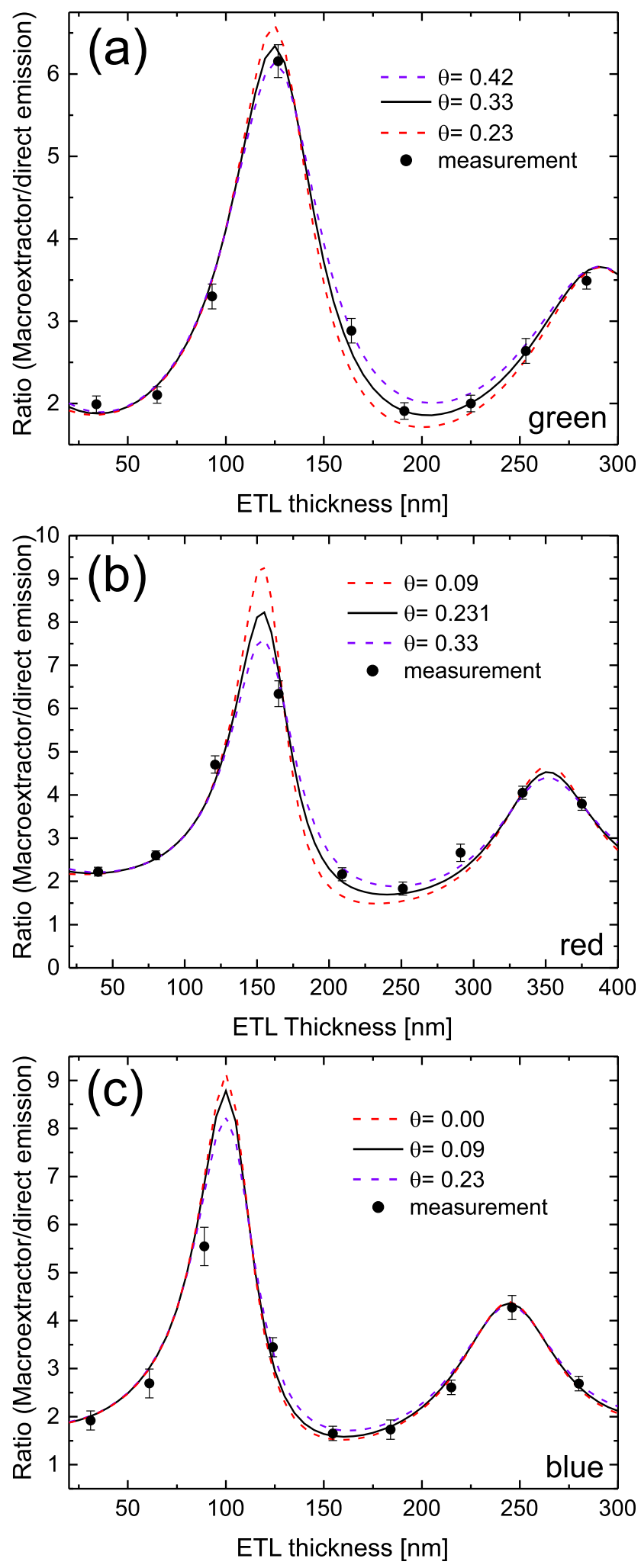

FIG. 4. Measured EQE ratio for (a) the green, (b) red, and (c) blue emitting system for a current density of $100 \mathrm{~mA} / \mathrm{cm}^{2}, 1 \mathrm{~mA} / \mathrm{cm}^{2}$, and $10 \mathrm{~mA} / \mathrm{cm}^{2}$, respectively (dots). The black lines represent the simulation for an emitter orientation of (a) $\theta=0.33$, (b) $\theta=0.23$, and (c) $\theta=0.09$. Additionally, the upper and lower limits for the orientation factors for the different emitting systems are displayed as dashed violet and red lines, respectively. The raw data of the here used measured EQE values of all three OLEDs are given in the supplementary information. ${ }^{26}$

orientations, which can be regarded as upper and lower limits for $\theta$, are displayed in the figures, too. It can clearly be seen that the other simulations cannot fit the measured data 
points in the same way, but they could be acceptable if some uncertainties in the ETL layer thickness and/or the measured EQE values appear, in particular, for the cavity minimum of the devices, which is sensitive to horizontal orientation of the transition dipole moments. Unfortunately, data points right in the cavity minimum are missing for the red and blue emitting system. Hence, an additional EQE analysis using data in the second cavity maximum of the devices is presented in the supplementary material, ${ }^{26}$ which clearly confirms the presented results. Note that this method only works reliably, if the layer thicknesses can be determined after the evaporation process in an accurate way and if the EQE measurements are performed with appropriate macroextractors and calibrated integrating spheres. Nevertheless, the presented analysis of the three different types of emitting systems can still be seen as convincing proof of concept that the emitter orientation can principally be extracted from simple EQE measurements.

In conclusion, we have presented a complementary and simple method to the previously published angular dependent emission pattern analysis ${ }^{5,6}$ for determining the orientation of the transition dipole moments of the emitting species in organic light-emitting guest/host systems. It is based on investigations of a set of OLEDs comprising different layer thicknesses via simple external quantum efficiency measurements using not only the direct emission but also extracting the captured substrate modes of the devices with a half sphere macroextractor lens. This clearly demonstrates the importance of taking substrate mode emission into account for consistent efficiency analysis of organic light-emitting diodes comprising non-isotropic emitter orientation. Furthermore, the anisotropy factors of three different emitting systems, exhibiting isotropic, slightly horizontal, and almost completely horizontal alignment of the emissive dipole moments are verified and compared to previously published results. The method does not require knowledge of the radiative exciton fraction or the emitter quantum efficiency and is independent of the applied current density.

We acknowledge financial support by the German Federal Ministry of Education and Research (BMBF) under the Contract Nos. 13N10474 and 13N12240, namely, projects "TOPAS 2012" and "OLYMP," respectively. We also acknowledge financial support by the Deutsche
Forschungsgemeinschaft (Contract No. BR 1728/13-1) as well as by the Bayerische Forschungsstiftung (C. Mayr).

${ }^{1}$ C. W. Tang and S. A. VanSlyke, Appl. Phys. Lett. 51, 913 (1987).

${ }^{2}$ S.-Y. Kim, W.-I. Jeong, C. Mayr, Y.-S. Park, K.-H. Kim, J.-H. Lee, C.-K. Moon, W. Brütting, and J.-J. Kim, Adv. Funct. Mater. 23, 3896 (2013).

${ }^{3}$ K.-H. Kim, C.-K. Moon, J.-H. Lee, S.-Y. Kim, and J.-J. Kim, Adv. Mater. 26(23), 3844 (2014).

${ }^{4}$ Commercial white OLEDs are offered since 2010, e.g., by Osram and Philips.

${ }^{5}$ M. Flämmich, M. C. Gather, N. Danz, D. Michaelis, A. H. Bräuer, K.

Meerholz, and A. Tünnermann, Org. Electron. 11, 1039 (2010).

${ }^{6}$ J. Frischeisen, D. Yokoyama, C. Adachi, and W. Brütting, Appl. Phys. Lett. 96, 073302 (2010).

${ }^{7}$ T. Tsutsui, E. Aminaka, C. P. Lin, and D. U. Kim, Philos. Trans. Soc. A 355, 801 (1997).

${ }^{8}$ M. A. Baldo, D. F. O’Brien, Y. You, A. Shoustikov, S. Sibley, M. E. Thompson, and S. R. Forrest, Nature 395, 151 (1998).

${ }^{9}$ J. S. Wilson, A. S. Dhoot, A. J. A. B. Seeley, M. S. Khan, A. Kohler, and R. H. Friend, Nature 413, 828 (2001).

${ }^{10}$ Y. Sun, N. C. Giebink, H. Kanno, B. Ma, M. E. Thompson, and S. R. Forrest, Nature 440, 908 (2006).

${ }^{11}$ Y. Luo and H. Aziz, Adv. Funct. Mater. 20, 1285 (2010).

${ }^{12}$ D. Yokoyama, Y. Park, B. Kim, S. Kim, Y.-J. Pu, J. Kido, and J. Park, Appl. Phys. Lett. 99, 123303 (2011).

${ }^{13}$ Y.-J. Pu, G. Nakata, F. Satoh, H. Sasabe, D. Yokoyama, and J. Kido, Adv. Mater. 24, 1765 (2012).

${ }^{14}$ H. Sternlicht, G. C. Nieman, and G. W. Robinson, J. Chem. Phys. 38, 1326 (1963).

${ }^{15}$ A. Endo, K. Sato, K. Yoshimura, T. Kai, A. Kawada, H. Miyazaki, and C. Adachi, Appl. Phys. Lett. 98, 083302 (2011).

${ }^{16}$ H. Uoyama, K. Goushi, K. Shizu, H. Nomura, and C. Adachi, Nature 492, 234 (2012).

${ }^{17}$ T. Nakagawa, S.-Y. Ku, K.-T. Wong, and C. Adachi, Chem. Commun. 48, 9580 (2012).

${ }^{18}$ T. D. Schmidt, D. S. Setz, M. Flämmich, J. Frischeisen, D. Michaelis, C. Mayr, A. F. Rausch, T. Wehlus, B. J. Scholz, T. C. G. Reusch, N. Danz, and W. Brütting, Appl. Phys. Lett. 103, 093303 (2013).

${ }^{19}$ C. Mayr, S. Y. Lee, T. D. Schmidt, T. Yasuda, C. Adachi, and W. Brütting, "Efficiency Enhancement of Organic Light-Emitting Diodes Incorporating a Highly Oriented Thermally Activated Delayed Fluorescence Emitter," Adv. Funct. Mater. (published online).

${ }^{20}$ T. D. Schmidt, M. Flämmich, B. J. Scholz, D. Michaelis, C. Mayr, N. Danz, and W. Brütting, Proc. SPIE 8435, 843513 (2012).

${ }^{21}$ T. D. Schmidt, D. S. Setz, M. Flämmich, J. Frischeisen, D. Michaelis, B. C. Krummacher, N. Danz, and W. Brütting, Appl. Phys. Lett. 99, 163302 (2011).

${ }^{22}$ T. Schmidt, B. Scholz, C. Mayr, and W. Brütting, IEEE J. Sel. Top. Quantum Electron. 19, 1 (2013).

${ }^{23}$ M. Flämmich, J. Frischeisen, D. S. Setz, D. Michaelis, B. C. Krummacher, T. D. Schmidt, W. Brütting, and N. Danz, Org. Electron. 12, 1663 (2011).

${ }^{24}$ W. Brütting, J. Frischeisen, T. D. Schmidt, B. J. Scholz, and C. Mayr, Phys. Status Solidi A 210, 44 (2013).

${ }^{25}$ D. S. Setz, T. D. Schmidt, M. Flämmich, S. Nowy, J. Frischeisen, B. C. Krummacher, T. Dobbertin, K. Heuser, D. Michaelis, N. Danz, W. Brütting, and A. Winnacker, J. Photon. Energy 1, 011006 (2011).

${ }^{26}$ See supplementary material at http://dx.doi.org/10.1063/1.4891680 for the investigation of the transition dipole orientation focusing on the second cavity maximum. 